\title{
Clinical Diagnostic Criteria of Familial Hypercholesterolemia \\ - A Comparison of the Japan Atherosclerosis Society and Dutch Lipid Clinic Network Criteria -
}

\author{
Hayato Tada, MD; Hirofumi Okada, MD; Akihiro Nomura, MD; \\ Soichiro Usui, MD; Kenji Sakata, MD; Atsushi Nohara, MD; \\ Masakazu Yamagishi, MD; Masayuki Takamura, MD; Masa-aki Kawashiri, MD
}

\begin{abstract}
Background: This study is aimed to compare the efficacy of the 2017 Japan Atherosclerosis Society (JAS) familial hypercholesterolemia (FH) criteria, which focuses on only 3 essential clinical manifestations, with that of Dutch Lipid Clinic Network (DLCN) FH criteria, which adopts a scoring system of multiple elements.
\end{abstract}

\begin{abstract}
Methods and Results: A total of 680 Japanese dyslipidemic participants (51\% men) were enrolled between 2006 and 2018 , all of whom had full evaluations of low-density lipoprotein (LDL) cholesterol, Achilles tendon X-rays, family history records, and genetic analysis of $\mathrm{FH}$-associated genes ( $L D L R, A P O B$, and PCSK9). Predictive values for the existence of $\mathrm{FH}$ mutations by both clinical criteria were evaluated. Overall, $173 \mathrm{FH}$ patients were clinically diagnosed by using the $2017 \mathrm{JAS}$ criteria and 100, 57, 156, and 367 subjects were also diagnosed as having definite, probable, possible, and unlikely FH by the DLCN FH criteria, respectively. The positive and negative likelihood ratio predicting the presence of $\mathrm{FH}$ mutations by using the $2017 \mathrm{JAS}$ FH criteria were 19.8 and 0.143 , respectively; whereas, using the DLCN criteria of definite, probable, and possible $\mathrm{FH}$, the ratios were 29.2 and $0.489,9.70$ and 0.332 , and 3.43 and 0.040 , respectively.
\end{abstract}

Conclusions: Among Japanese patients, the JAS 2017 FH criteria is considered superior to diagnose FH mutation-positive patients and simultaneously rule out FH mutation-negative patients compared with the DLCN FH criteria.

Key Words: Achilles tendon thickness; Familial hypercholesterolemia; Genetic analysis; Low-density lipoprotein receptor; Proprotein convertase subtilisin/kexin type 9

$\mathbf{F}$ amilial hypercholesterolemia (FH; OMIM \#143890) is characterized by a clinical triad of primary hyperlow-density lipoprotein (LDL)-cholesterolemia, tendon xanthomas, and premature coronary artery disease (CAD). ${ }^{1}$ Although the cause of FH is known to be deleterious mutations in genes correlated with the LDL receptor pathway, including $L D L$ receptor $(L D L R)$, apolipoprotein $B(A P O B)$, and proprotein convertase subtilisin/kexin type 9 (PCSK9), and accumulation of LDL-raising singlenucleotide polymorphisms, ${ }^{2}$ genetic testing is seldom utilized in clinical settings. ${ }^{3}$ In contrast, various clinical diagnostic criteria for $\mathrm{FH}$ worldwide exists, such as the Dutch Lipid Clinical Network (DLCN) FH criteria, ${ }^{4}$ the Simon-Bloom diagnostic criteria, ${ }^{5}$ Make Early Diagnosis to Prevent Early Death (MEDPED), ${ }^{6}$ and the 2017 Japan Atherosclerosis Society (JAS) FH criteria. ${ }^{7}$ Of those, the

\section{Editorial p 898}

DLCN scoring system is commonly utilized. The disadvantages of the DLCN FH criteria are: complicated calculation of the scores for each category, and a lack of quantitative definition of tendon xanthomas. In contrast, the 2017 JAS FH criteria are simple, quantifiable, and easy to utilize; FH is diagnosed if the following $\geq 2$ criteria are fulfilled: (1) LDL cholesterol $\geq 4.65 \mathrm{mmol} / \mathrm{L}$; (2) tendon xanthomas is on the dorsal side of the hands, elbows, or knees or Achilles tendon hypertrophy or xanthoma tuberosum; and (3) a family history of $\mathrm{FH}$ or premature CAD within second-degree relatives. ${ }^{7}$ An Achilles tendon thickness $\geq 9.0 \mathrm{~mm}$ is to be validated using X-rays. ${ }^{8}$ Nonetheless, no prior study specifically compares the efficacy of

Received August 27, 2020; revised manuscript received September 20, 2020; accepted September 26, 2020; J-STAGE Advance Publication released online December 3, 2020 Time for primary review: 16 days

Department of Cardiovascular Medicine, Graduate School of Medical Sciences, Kanazawa University, Kanazawa (H.T., H.O., A. Nomura, S.U., K.S., M.T., M.K.); Department of Genetics, Ishikawa Prefectural Central Hospital, Kanazawa (A. Nohara); and Osaka University of Human Sciences, Settsu (M.Y.), Japan

Mailing address: Masa-aki Kawashiri, MD, Department of Cardiovascular Medicine, Graduate School of Medical Sciences, Kanazawa University, 13-1 Takara-machi, Kanazawa 920-8641, Japan. E-mail: mk@med.kanazawa-u.ac.jp

All rights are reserved to the Japanese Circulation Society. For permissions, please e-mail: cj@j-circ.or.jp

ISSN-1346-9843 


\begin{tabular}{|c|c|c|c|c|}
\hline \multirow{2}{*}{ Variable } & \multirow{2}{*}{$\begin{array}{c}\text { All } \\
(n=680)\end{array}$} & \multicolumn{2}{|c|}{ CAD } & \multirow{2}{*}{$P$ value } \\
\hline & & Yes $(n=145)$ & No $(n=535)$ & \\
\hline Age (years) & $50 \pm 18$ & $61 \pm 13$ & $47 \pm 18$ & $<2 \times 10^{-16}$ \\
\hline Male & $344(51)$ & $104(72)$ & $240(45)$ & $1.3 \times 10^{-8}$ \\
\hline Body weight (kg) & $62 \pm 14$ & $67 \pm 14$ & $61 \pm 14$ & $2.8 \times 10^{-6}$ \\
\hline Hypertension & $247(36)$ & $115(79)$ & $132(25)$ & $<2 \times 10^{-16}$ \\
\hline Diabetes & $125(18)$ & $53(37)$ & $72(13)$ & $4.2 \times 10^{-10}$ \\
\hline Smoking & $242(36)$ & $111(77)$ & $131(24)$ & $<2 \times 10^{-16}$ \\
\hline Total cholesterol (mmol/L) & $6.83 \pm 78$ & $7.01 \pm 1.91$ & $6.59 \pm 2.04$ & $9.6 \times 10^{-4}$ \\
\hline Triglycerides (mmol/L) & $1.32[0.88-2.05]$ & $1.24[0.84-2.04]$ & $1.45[1.13-2.05]$ & 0.08 \\
\hline HDL cholesterol (mmol/L) & $1.37 \pm 0.44$ & $1.16 \pm 0.31$ & $1.42 \pm 0.47$ & $<2 \times 10^{-16}$ \\
\hline LDL cholesterol (mmol/L) & $4.53 \pm 1.86$ & $4.63 \pm 1.84$ & $4.34 \pm 1.89$ & $3.8 \times 10^{-4}$ \\
\hline Family history ${ }^{\dagger}$ & $180(26)$ & $28(19)$ & $152(28)$ & 0.04 \\
\hline FH mutation & $175(26)$ & $27(19)$ & $148(28)$ & 0.04 \\
\hline Achilles tendon thickness $(\mathrm{mm})$ & $7.2 \pm 3.0$ & $7.9 \pm 3.6$ & $7.0 \pm 2.8$ & 0.008 \\
\hline
\end{tabular}

Data are presented as mean $\pm \mathrm{SD}, \mathrm{n}(\%)$ or mean $[\mathrm{Cl}]$. CAD, coronary artery disease; $\mathrm{FH}$, familial hypercholesterol-

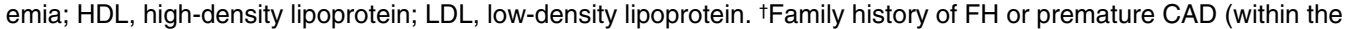
patient's second-degree relatives) according to the Japan Atherosclerosis Society (JAS) criteria.

2017 JAS FH criteria with the DLCN FH criteria. This study aims to compare the predicting value of both $\mathrm{FH}$ diagnostic criteria to predict the presence of pathogenic mutations of FH among Japanese dyslipidemic patients.

\section{Methods}

\section{Study Population}

A total of 1,981 patients with a measurement of Achilles tendon thickness suspecting FH at Kanazawa University Hospital between April 2006 and March 2018 were eligible to participate in the study. Screening did not include 1,092 patients due to a lack of lipid profiles and/or genetic analysis, 6 with homozygous or compound heterozygous $\mathrm{FH}, 1$ with autosomal recessive hypercholesterolemia, and 202 with a history of lipid-lowering therapy. A cohort of 680 participants with a mean age of $50 \pm 18$ years was included in this retrospective analysis. Overall, 344 men $(51 \%)$ and 145 women $(21 \%)$ had a history of CAD. The baseline data included a medical history review, a physical examination, and a blood draw. Most study participants were inpatients, allowing the acquisition of fasting blood samples.

\section{Genetic Analysis}

Genomic DNA was isolated from peripheral white blood cells, and polymerase chain reaction assays were conducted for clinically diagnosed FH participants following standard procedures. The exome regions of 21 dyslipidemiarelated genes with Mendelian inheritance, such as $3 \mathrm{FH}$ genes ( $L D L R, A P O B$, and PCSK9), were sequenced. The pathogenicity of the variants was determined using allele frequency, in silico analysis, and Clinvar (https://www. clinicalgenome.org/data-sharing/clinvar), as described previously. ${ }^{9}$

\section{Ethical Considerations}

The study was approved by the Ethics Committee of Kanazawa University and was conducted following the ethical standards of the responsible committee on human experimentation (institutional and national) and the Helsinki Declaration of 1975, as revised in 2008. The study participants provided informed consent for genetic analysis prior to inclusion.

\section{Biochemical Analysis}

Blood samples were collected following an overnight fasting, and serum total cholesterol, triglycerides, and highdensity lipoprotein (HDL) cholesterol were assayed enzymatically using an autoanalyzer (Qualigent, Sekisui Medical, Tokyo, Japan).$^{10}$ If serum levels of triglyceride were $<4.5 \mathrm{mmol} / \mathrm{L}$, then those of LDL cholesterol were measured using the Friedewald equation; otherwise, it was determined enzymatically. The initial data were acquired before the introduction of lipid-lowering treatments.

\section{Clinical Evaluation}

Smoking was defined as having any habitual smoking during the participants' lives. Achilles tendon thickness was evaluated with the use of X-ray, and Achilles tendon at the thickest part ( $\geq 9.0 \mathrm{~mm}$ as determined by X-ray) has been defined. Hypertension was defined as a systolic blood pressure of $\geq 140 \mathrm{mmHg}$ and/or a diastolic blood pressure of $\geq 90 \mathrm{mmHg}$ or the use of antihypertensive medications. Coexisting diabetes was defined as described by the Japan Diabetes Society or by the use of diabetes medications. CAD was defined by the presence of angina pectoris, myocardial infarction, or severe stenosis of the coronary arteries identified either on an angiogram or by computed tomography. ${ }^{11}$

\section{Assessment of Clinical Criteria for FH}

We assessed if the patients met each of the clinical diagnostic criteria for FH (JAS and DLCN), independently. The 2017 JAS criteria include 3 clinical manifestations, and patients are diagnosed as having $\mathrm{FH}$ if the following $\geq 2$ criteria are fulfilled: (1) LDL cholesterol $\geq 4.65 \mathrm{mmol} / \mathrm{L}$; (2) tendon xanthomas are present on the dorsal side of the hands, elbows, or knees or if the patients have Achilles tendon hypertrophy (an Achilles tendon thickness $\geq 9.0 \mathrm{~mm}$ on X-rays) or xanthoma tuberosum; and (3) a family history of FH or premature CAD within second-degree relatives. The DLCN FH criteria adopt a scoring system, 


\begin{tabular}{|c|c|c|c|c|c|c|c|}
\hline \multirow[b]{2}{*}{ Variable } & \multirow[b]{2}{*}{$\underset{(n=680)}{\text { All }}$} & \multicolumn{2}{|c|}{ JAS 2017 FH criteria } & \multicolumn{4}{|c|}{ DLCN FH criteria } \\
\hline & & $\begin{array}{l}\text { Positive } \\
(n=173)\end{array}$ & $\begin{array}{c}\text { Negative } \\
(n=507)\end{array}$ & $\begin{array}{l}\text { Definite } \\
(n=100)\end{array}$ & $\begin{array}{c}\text { Probable } \\
(n=57)\end{array}$ & $\begin{array}{c}\text { Possible } \\
(n=156)\end{array}$ & $\begin{array}{l}\text { Unlikely } \\
(\mathrm{n}=367)\end{array}$ \\
\hline Age (years) & $50 \pm 18$ & $40 \pm 16$ & $53 \pm 17$ & $43 \pm 14$ & $45 \pm 19$ & $46 \pm 18$ & $54 \pm 17$ \\
\hline Male & $344(51)$ & $72(42)$ & $272(54)$ & $47(47)$ & $27(47)$ & $84(54)$ & $186(51)$ \\
\hline Body weight (kg) & $62 \pm 14$ & $60 \pm 14$ & $63 \pm 14$ & $62 \pm 14$ & $63 \pm 19$ & $62 \pm 14$ & $62 \pm 13$ \\
\hline Hypertension & 247 (36) & $36(21)$ & $211(42)$ & $23(23)$ & $21(37)$ & $44(28)$ & $159(43)$ \\
\hline Diabetes & $125(18)$ & $12(7)$ & $113(22)$ & $7(7)$ & $12(21)$ & $16(10)$ & $90(25)$ \\
\hline Smoking & $242(36)$ & $46(27)$ & 196 (39) & $32(32)$ & $23(40)$ & $52(33)$ & $135(37)$ \\
\hline Total cholesterol (mmol/L) & $6.83 \pm 2.02$ & $8.69 \pm 1.81$ & $6.15 \pm 1.60$ & $9.23 \pm 1.89$ & $7.71 \pm 2.28$ & $7.55 \pm 1.19$ & $5.66 \pm 1.37$ \\
\hline Triglycerides (mmol/L) & $\begin{array}{c}1.32 \\
{[0.88-2.05]}\end{array}$ & $\begin{array}{c}1.08 \\
{[0.77-1.63]}\end{array}$ & $\begin{array}{c}1.40 \\
{[0.94-2.18]}\end{array}$ & $\begin{array}{c}1.16 \\
{[0.87-1.56]}\end{array}$ & $\begin{array}{c}1.23 \\
{[0.77-1.74]}\end{array}$ & $\begin{array}{c}1.35 \\
{[0.88-1.95]}\end{array}$ & $\begin{array}{c}1.39 \\
{[0.93-2.30]}\end{array}$ \\
\hline HDL cholesterol (mmol/L) & $1.37 \pm 0.44$ & $1.40 \pm 0.39$ & $1.37 \pm 0.47$ & $1.37 \pm 0.41$ & $1.37 \pm 0.39$ & $1.42 \pm 0.44$ & $1.34 \pm 0.47$ \\
\hline LDL cholesterol (mmol/L) & $4.53 \pm 1.86$ & $6.54 \pm 1.71$ & $3.83 \pm 1.34$ & $7.03 \pm 1.81$ & $5.59 \pm 2.04$ & $5.35 \pm 0.93$ & $3.34 \pm 0.98$ \\
\hline CAD (\%) & $145(21)$ & $30(17)$ & $115(23)$ & $23(23)$ & $12(21)$ & $37(24)$ & $73(20)$ \\
\hline FH mutation & $175(26)$ & $151(87)$ & $24(5)$ & $91(91)$ & $30(53)$ & $49(31)$ & $5(1)$ \\
\hline Achilles tendon thickness $(\mathrm{mm})$ & $7.2 \pm 3.0$ & $10.3 \pm 4.1$ & $6.1 \pm 1.4$ & $12.4 \pm 3.9$ & $8.9 \pm 2.9$ & $6.3 \pm 1.2$ & $5.9 \pm 1.1$ \\
\hline
\end{tabular}

Data are presented as mean \pm SD, $n$ (\%) or mean [Cl]. DLCN, Dutch Lipid Clinic Network. Other abbreviations as in Table 1.

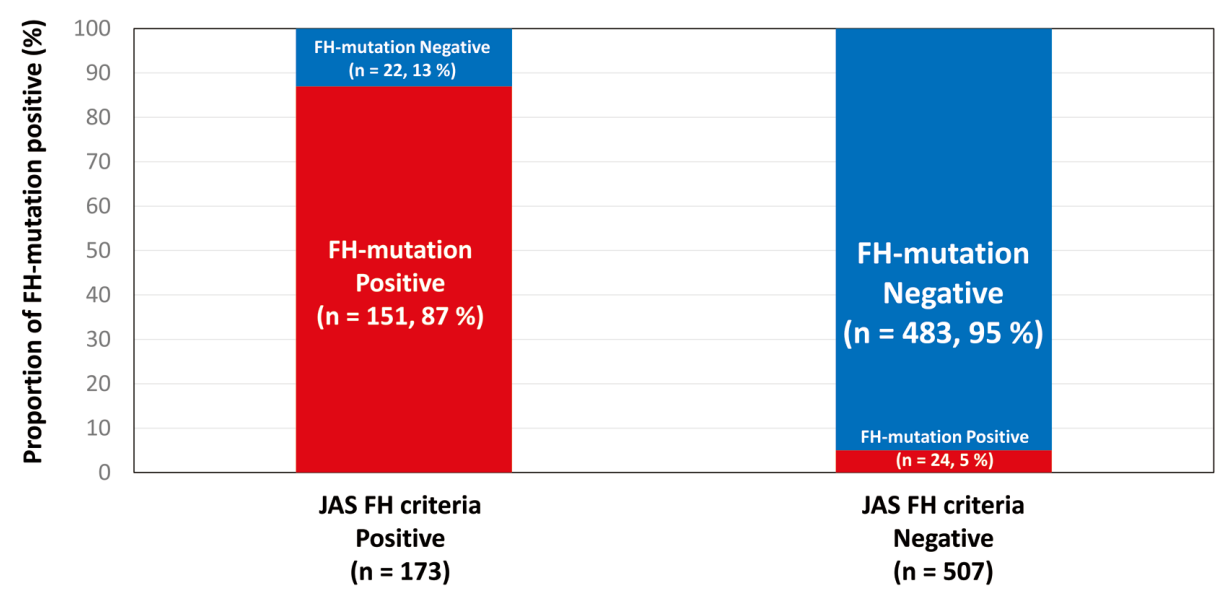

Figure 1. Proportions of FH mutation-positive patients according to the JAS $2017 \mathrm{FH}$ criteria. The left bar includes the patients categorized as positive by the JAS $2017 \mathrm{FH}$ criteria. The right bar includes the patients categorized as negative by the JAS 2017 FH criteria. Red indicates FH mutation positive, and blue indicates FH mutation negative. FH, familial hypercholesterolemia; JAS, Japan Atherosclerosis Society.

including family history, clinical history, physical examination, and LDL cholesterol levels. Patients are categorized as definite-FH (DLCN score $>8$ ), probable-FH (DLCN score 6-8), possible-FH (DLCN score 3-5), and unlikelyFH (DLCN score <3). ${ }^{\mathbf{2}}$ Sensitivity, specificity, positive predictive value (PPV), negative predictive value (NPV), positive likelihood ratio (PLR), and negative likelihood ratio (NLR) to predict $\mathrm{FH}$ mutation are calculated for both the JAS 2017 criteria and the DLCN criteria. The predictive values of DLCN were calculated as a sum of the subjects in the category and its stricter categories vs. the rest of the subjects; for example, the predictive values of probable-FH were calculated by comparison between definite plus probable-FH and possible plus unlikely-FH.

\section{Statistical Analysis}

Categorical variables were described as percentages and compared by using Fisher's exact test or the chi-squared test, whichever was suitable. Continuous variables with a normal distribution were reported as means \pm standard deviation (SD). Variables that were not normally distributed were described as medians and interquartile range (IQR). Mean values of continuous variables were compared with the Student's t-test for independent data; and median values were compared with the non-parametric Wilcoxon Mann-Whitney rank sum test or the chi-squared test for categorical variables with Fisher's post-hoc test. Multivariate logistic regression analysis was performed and included all variables to determine the factors associated with CAD. The statistical analysis was performed using R statistics (https://www.r-project.org). P values of $<0.05$ were considered statistically significant. 


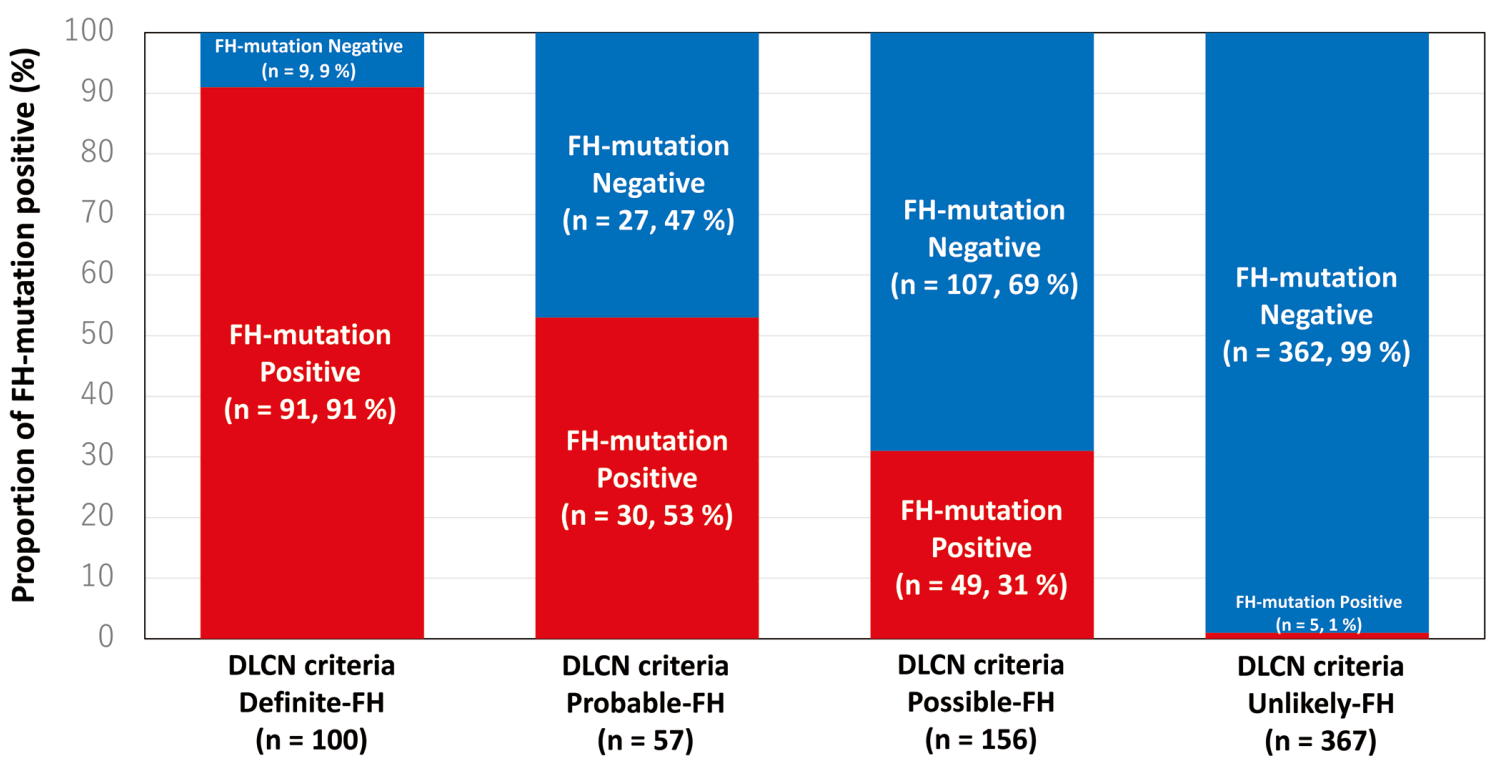

Figure 2. Proportions of FH mutation-positive patients according to the DLCN FH criteria. Red indicates FH mutation positive, and blue indicates FH mutation negative. FH, familial hypercholesterolemia; DLCN, Dutch Lipid Clinic Network.

\begin{tabular}{|lcccccc|}
\hline \multicolumn{2}{|l}{ Table 3. Predictive Value of Each Clinical Criterion } & & & & \\
$\quad$ Criteria & Sensitivity & Specificity & PPV & NPV & PLR & NLR \\
JAS 2017 FH criteria & 0.863 & 0.956 & 0.873 & 0.953 & 19.806 & 0.143 \\
DLCN & & & & & & \\
$\quad$ Definite-FH & 0.520 & 0.982 & 0.910 & 0.855 & 29.178 & 0.489 \\
Probable-FH & 0.691 & 0.929 & 0.771 & 0.897 & 9.699 & 0.332 \\
Possible-FH & 0.971 & 0.717 & 0.543 & 0.986 & 3.431 & 0.040 \\
\hline
\end{tabular}

NLR, negative likelihood ratio; NPV, negative predictive value; PLR, positive likelihood ratio; PPV, positive predictive value. Other abbreviations as in Tables 1,2. The predictive values of DLCN were calculated as the sum of the subjects in the category and its stricter categories vs. the rest of the subjects; for example, the predictive values of probable-FH were calculated by comparison between definite plus probable-FH and possible plus unlikely-FH.

\section{Results}

\section{Participant Characteristics}

The clinical characteristics of the study participants are shown in Table 1. Based on the fact that CAD is the most important complication of FH, we showed baseline characteristics according to CAD status. The mean age was $50 \pm 18$ years, and the mean LDL cholesterol was $4.53 \pm 1.86 \mathrm{mmol} / \mathrm{L}$. $\mathrm{FH}$-associated mutations were identified in 175 of the 680 participants (26\%), 156 with $L D L R$ mutations, and 19 with displayed PCSK 9 mutations (Supplementary Table 1). As shown in Table 1, there were significant differences in the presence of mutations in participants with and without CAD.

\section{Clinical Characteristics According to the JAS 2017 and the DLCN FH Criteria}

As shown in Table 2, patients with a positive status of $\mathrm{FH}$ as determined by either clinical criteria tended to be younger; have less probability to have diabetes, higher LDL cholesterol, and less triglycerides; and displayed a thicker Achilles tendon than with patients with negative clinical status.

\section{Predictive Value for FH Mutation Using the JAS 2017 and the DLCN FH Criteria}

We assessed the predictive values for the presence of $\mathrm{FH}$ mutation using the JAS 2017 and the DLCN FH criteria. With the use of the JAS 2017 FH criteria, we identified 173 $\mathrm{FH}$ and 507 non-FH patients. Among those $173 \mathrm{FH}$ patients diagnosed by using the JAS $2017 \mathrm{FH}$ criteria, we found 151 FH mutation-positive patients $(87 \%)$. In contrast, we found 24 mutation-positive patients among 507 non-FH patients $(5 \%)$ by using the JAS 2017 FH criteria (Figure 1). And, with the use of the DLCN FH criteria, we identified 100 definitive-FH patients, 57 probable-FH patients, 156 possible-FH patients, and 367 unlikely-FH patients. Among those patients, FH mutation was found in 91 (91\%), 30 $(53 \%), 49(31 \%)$, and $5(1 \%)$ patients, respectively (Figure 2). Sensitivity, specificity, PPV, NPV, PLR, and NLR for predicting FH mutations by using the JAS 2017 and the DLCN FH criteria are shown in Table 3.

Moreover, a receiver operating characteristic analysis suggested that the best cut-off value predicting FH-mutation as $7.0 \mathrm{~mm}$. We further evaluated predictive value of each clinical criterion in case the threshold of Achilles 


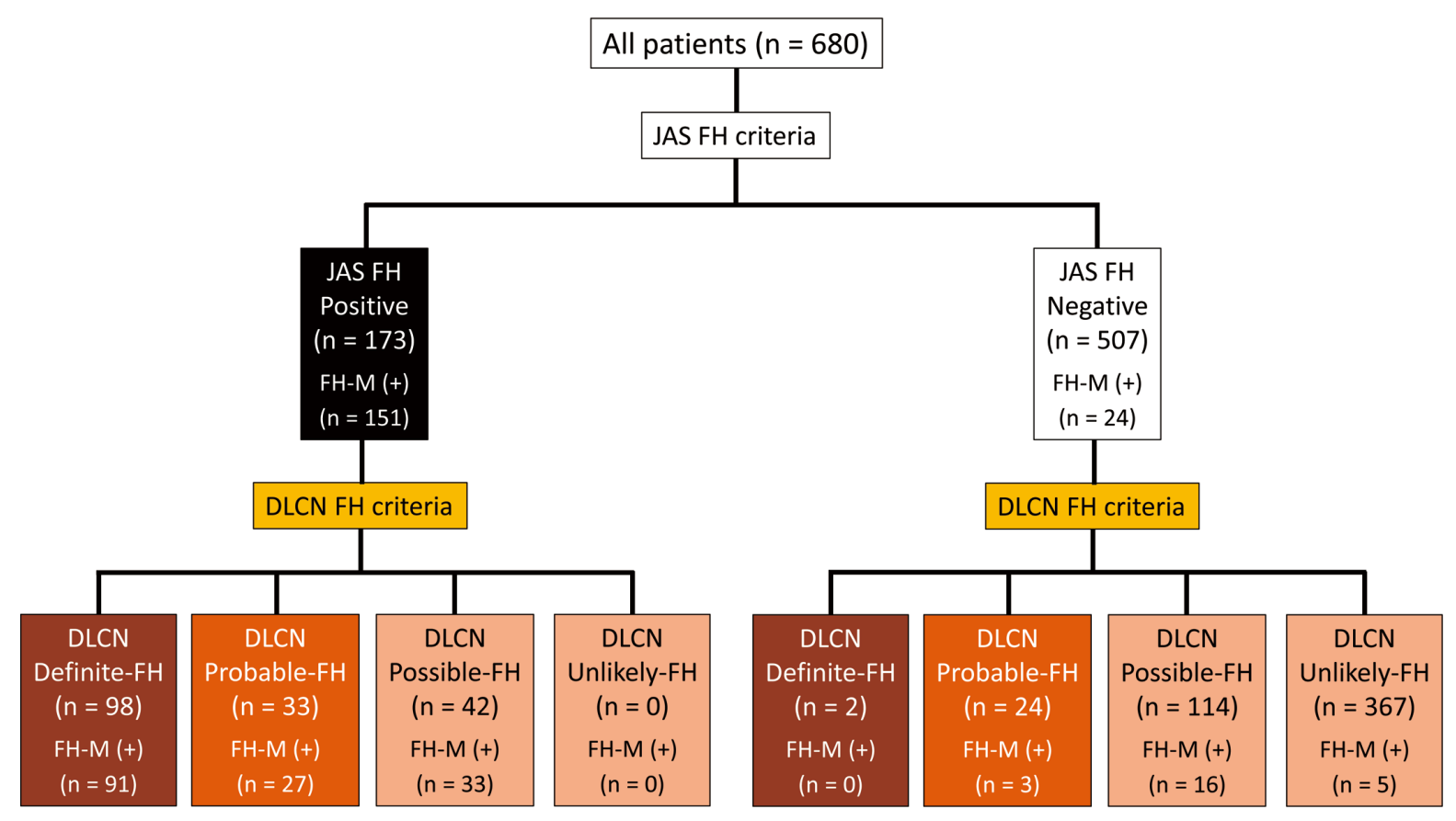

Figure 3. Reclassification chart from the JAS $2017 \mathrm{FH}$ criteria to the DLCN FH criteria. All patients were evaluated via the JAS $2017 \mathrm{FH}$ criteria at first and then re-evaluated via the DLCN FH criteria. The numbers of reclassified patients and FH mutation positive are shown. FH, familial hypercholesterolemia; JAS, Japan Atherosclerosis Society; DLCN, Dutch Lipid Clinic Network.

tendon thickness was changed from $\geq 9.0 \mathrm{~mm}$ to $\geq 7.0 \mathrm{~mm}$ (Supplementary Table 2). As expected, we observed higher sensitivity and lower specificity in both criteria.

\section{Reclassification of Clinical Diagnosis of FH Using the JAS 2017 and the DLCN FH Criteria}

We evaluated the reclassifications of clinical $\mathrm{FH}$ diagnosis using the JAS 2017 criteria and the DLCN FH criteria on Japanese dyslipidemic patients. Among $173 \mathrm{FH}$ patients diagnosed by using the JAS $2017 \mathrm{FH}$ criteria, we reclassified $98(57 \%), 33(19 \%), 42(24 \%)$, and $0(0 \%)$ patients into DLCN definite FH, probable $\mathrm{FH}$, possible $\mathrm{FH}$, and unlikely FH, respectively. Among those patients, we found 91, 27, 33, and 0 patients with FH mutation, respectively (Figure 3, Supplementary Figure).

In contrast, among 507 non-FH patients diagnosed by using the JAS 2017 FH criteria, we reclassified 2, 24, 114, and 367 patients into DLCN definite $\mathrm{FH}$, probable $\mathrm{FH}$, possible $\mathrm{FH}$, and unlikely $\mathrm{FH}$, respectively. Among those patients, we discovered $0,3,16$, and 5 patients with $\mathrm{FH}$ mutation, respectively (Figure 3, Supplementary Figure). Of note, 5 non-FH patients who were diagnosed both by using the JAS 2017 and the DLCN criteria possessed FH mutations.

\section{Factors Associated With CAD}

Multivariate logistic regression analysis revealed that classical risk factors, including age, male, hypertension, smoking, triglycerides, HDL cholesterol, FH-mutation, and Achilles tendon thickness $\geq 9.0 \mathrm{~mm}$ were significantly associated with CAD (Supplementary Table 3).

\section{Discussion}

In this study, we compared the clinical criteria of $\mathrm{FH}$ in Japan (the JAS 2017 FH criteria) to that of the Netherlands (the DLCN FH criteria) among Japanese dyslipidemic patients. We found that the JAS 2017 FH criteria, focusing only on 3 essential clinical manifestations, was definitively easy-to-handle and superior to rule out the subjects absent of FH mutations with considerable sensitivity compared with the DLCN FH criteria, which uses a complicated scoring system that consists of multiple elements. In contrast, the DLCN FH criteria have great advantages, such as: (1) less chance to be denied to have $\mathrm{FH}$, because of multiple clinical elements, especially by a specific factor of clinical history of premature CAD of the patients (367 patients by the DLCN unlikely-FH vs. 507 patients by the JAS 2017 non-FH), (2) low NLR of possible-FH based on the fact that 5 patients $(1 \%)$ turned out to be $\mathrm{FH}$ mutationpositive among 367 patients categorized as DLCN unlikely-FH, whereas 24 patients $(5 \%)$ turned out to be $\mathrm{FH}$ mutation-positive among 507 patients categorized as JAS 2017 non-FH; (3) genetic diagnosis is actually one of the diagnostic elements, thus the 5 non-FH patients clinically diagnosed by both the JAS 2017 and the DLCN FH criteria turned out to be definite-FH. From this view point, it would be better to incorporate genetic diagnosis in future FH diagnostic criteria.

It is worth noting that the JAS 2017 FH criteria did not account for the clinical history of premature CAD of the patients, which led to less chance to be denied to have $\mathrm{FH}$, especially among those with no clear family history. In this regard, there was an interesting study investigating the 
prevalence of $\mathrm{FH}$ among acute coronary syndrome patients using JAS 2017 FH criteria as well as DLCN FH criteria. ${ }^{13}$ In this study, Tanaka et al found that substantial proportions $(>10 \%)$ of acute coronary syndrome patients aged $<60$ years turned out to have $\mathrm{FH}$, as diagnosed by either criteria. Actually, the overall conclusion appears to be quite similar to ours, although the aim of their study was different from ours (the previous study investigated the prevalence of $\mathrm{FH}$ among acute coronary syndrome patients, and did not check genetic background). First, JAS 2017 FH criteria have big advantages in its simplicity without losing sensitivity and specificity. Second, the definition of xanthomas is arbitrary in DLCN FH criteria, thus, reproducibility of clinical diagnosis is difficult. Third, DLCN FH criteria have additional categories, including probable, and potential $\mathrm{FH}$, thus, the sensitivity when accounting for those categories may be better when using DLCN FH criteria than JAS 2017 FH criteria.

Recently, a consensus has been reached with regards to the prevalence of $\mathrm{FH}$ based on various systematic reviews and meta-analyses, proposing 1 in $~ 300$ among general populations worldwide. ${ }^{14-19}$ Its prevalence substantially increases among patients with ischemic heart disease $(\sim 10$ fold), premature ischemic heart disease ( 20 -fold), and severe hypercholesterolemia ( 23-fold). Various types of clinical diagnostic criteria for $\mathrm{FH}$ worldwide were noted, such as the DLCN FH criteria, the Simon Broome Diagnostic Criteria, the MEDPED diagnostic criteria, and the JAS FH diagnostic criteria. Few data exist that compare directly those diagnostic criteria, ${ }^{\mathbf{2 0}}$ and as far as we know, no prior study specifically compares the JAS 2017 with the DLCN FH criteria. We basically found that the JAS 2017 FH criteria work better than the DLCN FH criteria, in terms of superior specificity, NPV, and NLR with considerable sensitivity, PPV, and PLR. However, the threshold of Achilles tendon thickness can be lowered to some degree if we wish to increase the diagnostic rate of $\mathrm{FH}$.

The question of what is the definition of FH (e.g., is it a monogenic disorder correlated with elevated LDL, an inherited disorder correlated with elevated LDL, or hypercholesterolemia with xanthomas), is still unanswered. The prevention of CAD development by early diagnosis of $\mathrm{FH}$ should be the clinical objective. ${ }^{21}$ In this regard, the DLCN FH criteria have great advantages, which include "probable" and "possible" FH rather than quit the clinical diagnosis of $\mathrm{FH}$.

\section{Study Limitations}

The limitations of this study include its retrospective, cross-sectional, and observational design. Nonetheless, it included a large sample of the Japanese population, which adds to our understanding of FH across ethnicities. Second, the inclusion of participants whose Achilles tendon thickness had been measured for any reason could have instituted a selection bias. Third, genetic analysis was not conducted in all participants with Achilles tendon thickness measurements. Fourth, the mutation positive rate in this study was rather higher than that previously described $(60-80 \%) ;{ }^{7}$ there are 3 potential reasons for this phenomena: (1) the lack of clinical or genetic data from patients needed to assess both JAS and DLCN FH criteria were excluded. Accordingly, more intensively assessed patients who are more likely to have a pathogenic mutation tended to be included in this study; (2) we did not exclude cascadescreened patients in this study, thereby, potentially causing a higher mutation detection rate in this study; and (3) approximately one-third of the patients had the same nonsense mutation frequently found in the Hokuriku district of Japan, which also affects the higher mutation detection rate in this study.

\section{Conclusions}

Among Japanese patients, the JAS 2017 FH criteria focusing on only 3 essential clinical manifestations, is considerably superior in diagnosing $\mathrm{FH}$ mutation-positive patients and simultaneously rule out FH mutation-negative patients compared with the DLCN FH criteria.

\section{Acknowledgments}

The authors express their special thanks to Kazuko Honda and Sachio Yamamoto of Kanazawa University for their excellent technical assistance.

\section{Sources of Funding}

This work was supported by scientific research grants from the Ministry of Education, Science, and Culture of Japan (No. 16K19394, and 18K08064), Astellas Foundation for Research on Metabolic Disorders, ONO Medical Research Foundation, and Japanese Circulation Society.

\section{Disclosures}

The authors declare no conflicts of interest.

\section{IRB Information}

The present study was approved by Kanazawa University (Reference \# 2018-004).

\section{References}

1. Kawashiri M, Hayashi K, Konno T, Fujino N, Ino H, Yamagishi M. Current perspectives in genetic cardiovascular disorders: From basic to clinical aspects. Heart Vessels 2014; 29: 129-141.

2. Talmud PJ, Shah S, Whittall R, Futema M, Howard P, Cooper JA, et al. Use of low-density lipoprotein cholesterol gene score to distinguish patients with polygenic and monogenic familial hypercholesterolaemia: A case-control study. Lancet 2013; 381: $1293-1301$

3. Sturm AC, Knowles JW, Gidding SS, Ahmad ZS, Ahmed CD, Ballantyne CM, et al. Clinical genetic testing for familial hypercholesterolemia: JACC Scientific Expert Panel. J Am Coll Cardiol 2018; 72: 662-680.

4. Austin MA, Hutter CM, Zimmern RL, Humphries SE. Genetic causes of monogenic heterozygous familial hypercholesterolemia: A HuGE prevalence review. Am J Epidemiol 2004; 160: 407-420.

5. Scientific Steering Committee on behalf of the Simon Broome Register Group. Risk of fatal coronary heart disease in familial hypercholesterolaemia. BMJ 1991; 303: 893-896.

6. Williams RR, Hunt SC, Schumacher MC, Hegele RA, Leppert MF, Ludwig EH, et al. Diagnosing heterozygous familial hypercholesterolemia using new practical criteria validated by molecular genetics. Am J Cardiol 1993; 72: 171-176.

7. Harada-Shiba M, Arai H, Ishigaki Y, Ishibashi S, Okamura T, Ogura M, et al. Guidelines for diagnosis and treatment of familial hypercholesterolemia 2017. J Atheroscler Thromb 2018; 25: $751-770$.

8. Mabuchi H, Ito S, Haba T, Ueda K, Ueda R. Discrimination of familial hypercholesterolemia and secondary hypercholesterolemia by Achilles' tendon thickness. Atherosclerosis 1977; 28: $61-68$.

9. Tada H, Kawashiri MA, Nomura A, Teramoto R, Hosomichi $\mathrm{K}$, Nohara A, et al. Oligogenic familial hypercholesterolemia, LDL cholesterol, and coronary artery disease. J Clin Lipidol 2018; 12: 1436-1444.

10. Tada H, Kawashiri MA, Takata M, Matsunami K, Imamura A, Matsuyama M, et al. Infantile cases of sitosterolemia with novel mutations in the ABCG5 gene: Extreme hypercholesterolemia is 
exacerbated by breastfeeding. JIMD Rep 2015; 21: 115-122.

11. Tada H, Kawashiri MA, Yoshida T, Teramoto R, Nohara A, Konno T, et al. Lipoprotein(a) in familial hypercholesterolemia with proprotein convertase subtilisin/kexin type 9 (PCSK9) gainof-function mutations. Circ J 2016; 80: 512-518.

12. Nordestgaard BG, Chapman MJ, Humphries SE, Ginsberg HN, Masana L, Descamps OS, et al. Familial hypercholesterolaemia is underdiagnosed and undertreated in the general population: Guidance for clinicians to prevent coronary heart disease: Consensus statement of the European Atherosclerosis Society. Eur Heart J 2013; 34: 3478-3490.

13. Tanaka N, Teramoto T, Yokoyama S. Application of the Japanese guidelines for the diagnosis of familial hypercholesterolemia in general practice: It is to be validated in international harmonization. $J$ Atheroscler Thromb 2019; 26: 93-98.

14. Mabuchi H, Nohara A, Noguchi T, Kobayashi J, Kawashiri MA, Tada H, et al. Molecular genetic epidemiology of homozygous familial hypercholesterolemia in the Hokuriku district of Japan. Atherosclerosis 2011; 214: 404-407.

15. Benn M, Watts GF, Tybjærg-Hansen A, Nordestgaard BG. Mutations causative of familial hypercholesterolaemia: Screening of 98098 individuals from the Copenhagen General Population Study estimated a prevalence of 1 in 217. Eur Heart J 2016; 37: $1384-1394$.

16. de Ferranti SD, Rodday AM, Mendelson MM, Wong JB, Leslie LK, Sheldrick RC. Prevalence of familial hypercholesterolemia in the 1999 to 2012 United States National Health and Nutrition
Examination Surveys (NHANES). Circulation 2016; 133: 1067-1072.

17. Wald DS, Bestwick JP, Morris JK, Whyte K, Jenkins L, Wald NJ. Child-parent familial hypercholesterolemia screening in primary care. N Engl J Med 2016; 375: 1628-1637.

18. Beheshti SO, Madsen CM, Varbo A, Nordestgaard BG. Worldwide prevalence of familial hypercholesterolemia: Meta-analyses of 11 million subjects. J Am Coll Cardiol 2020; 75: 2553-2566.

19. Hu P, Dharmayat KI, Stevens CAT, Sharabiani MTA, Jones RS, Watts GF, et al. Prevalence of familial hypercholesterolemia among the general population and patients with atherosclerotic cardiovascular disease: A systematic review and meta-analysis. Circulation 2020; 141: 1742-1759.

20. Chan DC, Pang J, Hooper AJ, Bell DA, Bates TR, Burnett JR, et al. A comparative analysis of phenotypic predictors of mutations in familial hypercholesterolemia. J Clin Endocrinol Metab 2018; 103: $1704-1714$.

21. Tada H, Kawashiri MA, Nohara A, Inazu A, Mabuchi H, Yamagishi M. Impact of clinical signs and genetic diagnosis of familial hypercholesterolaemia on the prevalence of coronary artery disease in patients with severe hypercholesterolaemia. Eur Heart J 2017; 38: $1573-1579$.

\section{Supplementary Files}

Please find supplementary file(s);

http://dx.doi.org/10.1253/circj.CJ-20-0901 